\title{
Structural phase transition, optical and pyroelectric properties of lead-free single crystals
}

\author{
FANG BiJun $^{1 *}$, WANG Meng ${ }^{1}$, YUAN Ning Yi ${ }^{1}$, DING JianNing ${ }^{1,2}$, ZHAO Xiang Yong $^{3}$, \\ XU HaiQing ${ }^{3}$ \& LUO HaoSu ${ }^{3}$ \\ ${ }^{1}$ School of Materials Science and Engineering, Changzhou University, Changzhou 213164, China; \\ ${ }^{2}$ School of Materials Science and Engineering, Jiangsu University, Zhenjiang 212013, China; \\ ${ }^{3}$ Key Laboratory of Inorganic Function Material and Device, Chinese Academy of Sciences, Shanghai 201800, China
}

Received December 12, 2012; accepted February 21, 2013; published online August 15, 2013

0.14 at\% Mn-doped $0.95\left(\mathrm{Na}_{1 / 2} \mathrm{Bi}_{1 / 2}\right) \mathrm{TiO}_{3}-0.05 \mathrm{BaTiO}_{3}(0.95 \mathrm{NBT}-0.05 \mathrm{BT})$ lead-free single crystals were grown by a top-seeded solution growth method (TSSG). X-ray powder diffraction measurement showed that the as-grown single crystals exhibit rhombohedral perovskite structure with apparent distortion. With the increase of temperature, successive structural phase transitions occur in the Mn-doped 0.95NBT-0.05BT single crystals. After poling, apparent dielectric anomaly is induced accompanied by the increase of the character of diffuse phase transition and the decrease of the value of dielectric constant. The Mn-doped 0.95NBT-0.05BT single crystals exhibit complex domain structure, in which micro and macro domains coexist with statistically 4 $\mathrm{mm}$ symmetry. The Mn-doped 0.95NBT-0.05BT lead-free single crystals exhibit excellent piezoelectric and pyroelectric properties, which will lead to promising advance in piezoelectric and pyroelectric applications. The infrared-absorption band occurred around $630.6 \mathrm{~cm}^{-1}$ can be assigned to the "stretching" normal vibration of $\left[\mathrm{Na}_{1 / 2} \mathrm{Bi}_{1 / 2}\right] / \mathrm{Ti}-\mathrm{O}$ group. The three diffused Raman bands centered around 300,560 and $800 \mathrm{~cm}^{-1}$ can be attributed to $\mathrm{F} 2 \mathrm{~g}\left[\mathrm{TiO}_{6}\right]$ bending vibration, $\mathrm{A} 1 \mathrm{~g}\left[\mathrm{TiO}_{6}\right]$ stretching vibration and "soft mode" mixed by bending and stretching vibrations.

Mn-doped 0.95NBT-0.05BT single crystals, structural phase transition, domain configuration, electrical properties

Citation: $\quad$ Fang B J, Wang M, Yuan N Y, et al. Structural phase transition, optical and pyroelectric properties of lead-free single crystals. Chin Sci Bull, 2013, 58: 4064-4071, doi: 10.1007/s11434-013-5979-8

With the demand for sustainable development of environmental protection and human society, development of lead-free piezoelectric materials that can replace hazardous lead-based $\mathrm{Pb}\left(\mathrm{Zr}_{x} \mathrm{Ti}_{1-x}\right) \mathrm{O}_{3}$ (PZT) piezoelectric ceramics has been research hot in piezoelectric application fields [1-3]. Among lead-free piezoelectric materials, $(1-x)\left(\mathrm{Na}_{1 / 2} \mathrm{Bi}_{1 / 2}\right)$ $\mathrm{TiO}_{3}-x \mathrm{BaTiO}_{3}$ (NBT-BT) solid solutions attract great research attention due to their excellent piezoelectric performance [4,5]. Since piezoelectric materials existence in crystallographically engineered single crystals exhibit more excellent piezoelectric properties, much work has been undertaken on the growth of high-quality and large-size NBT-BT single crystals [6,7].

*Corresponding author (email: fangbj@ sohu.com; fangbj@cczu.edu.cn)
From the viewpoint of crystal chemistry, the extraordinarily enhanced piezoelectric properties of solid solution ferroelectrics are considered as correlating with the engineered ferroelectric domain configuration, the relaxor nature of the compositions around the morphotrophic phase boundary (MPB), and the polarization rotation via intermediate monoclinic or orthorhombic states [8-10]. As for the NBT-BT system, Takenaka et al. [4] determined its MPB composition locating at $x=0.06-0.07$, in which the NBT-BT single crystals with the compositions around the MPB exhibited large electric field-induced strain and excellent piezoelectric property [11]. However, the contributions of engineered domain structure, relaxor behavior nature and polarization rotation path on the MPB effects should be given more fundamental research. 
In this study, 0.14 at $\%$ Mn-doped $0.95\left(\mathrm{Na}_{1 / 2} \mathrm{Bi}_{1 / 2}\right) \mathrm{TiO}_{3}$ $0.05 \mathrm{BaTiO}_{3}(0.95 \mathrm{NBT}-0.05 \mathrm{BT})$ single crystals grown by a top-seeded solution growth method (TSSG) were investigated. This composition locates at the rhombohedral side around the MPB of the NBT-BT system [4], which tends to exhibit excellent piezoelectric property according to the engineered domain theory. Mn doping was adopted to decrease the relatively large conductivity and to solve the polarization difficulty of the $\left(\mathrm{Na}_{1 / 2} \mathrm{Bi}_{1 / 2}\right) \mathrm{TiO}_{3}$-based (NBT) materials induced by its large coercive field $E_{\mathrm{c}}$ and conductivity. The structural phase transition character, the influences of crystal orientation on optical property and of polarization on domain configuration and electrical properties were investigated, which will deepen the understanding of the structure-property relations and the origin of high piezoelectric response, and promote their practical applications.

\section{Experimental procedure}

The Mn-doped 0.95NBT-0.05BT single crystals used in this study were grown by the TSSG method as reported elsewhere [6]. The as-grown crystals exhibit dark brown color due to the Mn doping. Crystal powder and $3 \mathrm{~mm} \times 3 \mathrm{~mm} \times$ $0.5 \mathrm{~mm}$ crystal plates with pseudo-cubic [001], [110] and [111] orientations determined by X-ray diffractionmeter were prepared for crystal structure characterization and physical performances measurements.

Crystal structure of the Mn-doped 0.95NBT-0.05BT single crystals was investigated by X-ray diffractometry (XRD, Rigaku D/max-2500/PC X-ray diffractionmeter) using crystal powder, where structural phase transition of the crystal upon heating was measured by an SHT- 1500 2311B501 high-temperature apparatus attached to the XRD equipment. Specific heat per unit mass $C_{\mathrm{p}}$ upon heating was measured by differential scanning calorimetry (Pyris DSC 8500 ) between room temperature and $250^{\circ} \mathrm{C}$ in air at a heating temperature rate of $10^{\circ} \mathrm{C} / \mathrm{min}$ using sapphire as standard sample. Domain structure of the [001]-oriented Mn-doped 0.95NBT-0.05BT crystal plate was observed by Nikon eclipse 50iPOL polarizing microscope (Nikon Corporation, Japan) using transmission mode under crossed polarized-light, in which a QImaging MicroPublisher (Rohs, Canada) CCD was attached to obtain precision high-fidelity color imaging. Fourier transform infrared spectroscopy (FT-IR) was recorded by Thermo Nicolet Avatar 370DGTS FT-IR Spectrometer using the Mn-doped 0.95NBT-0.05BT crystal powder dispersed in pressed $\mathrm{KBr}$ disk in transmission mode (infrared-absorption spectrum) and using the Mn-doped 0.95NBT-0.05BT crystal plates with different orientations in reflection mode (infrared-reflection spectrum). Raman spectra were recorded at room temperature in back scattering geometry with Thermo Scientific DXR Raman Microscope using Ar-ion laser operated at $532 \mathrm{~nm}$.

For electrical properties measurements, both the large 3 $\mathrm{mm} \times 3 \mathrm{~mm}$ surfaces were coated by silver paste and fired in air at $600^{\circ} \mathrm{C}$ for $15 \mathrm{~min}$ to form robust electrodes. Bulk resistivity of the Mn-doped 0.95NBT-0.05BT crystal plates with different orientations was measured by Agilent 4339B High Resistance Meter (Agilent Technologies Japan Ltd. Component Test PGU-Kobe, Hyogo, Japan), in which an Agilent 16008B resistivity cell was attached via cable connection to set the samples. All the crystal planes were poled under an electric field of $4 \mathrm{kV} / \mathrm{mm}$ perpendicular to the 3 $\mathrm{mm} \times 3 \mathrm{~mm}$ surfaces at room temperature for $5 \mathrm{~min}$ in silicon oil. Dielectric property was measured by Turnkey broadband dielectric spectrometers Concept 42 (Novocontrol Technologies GmbH \& Co. KG, Germany). Pyroelectric coefficient of the Mn-doped 0.95NBT-0.05BT crystal plates with different orientations was measured by a computer-controlled PY2 pyroelectric measurement system. Piezoelectric property was measured by a ZJ-6A Berlincourt-type quasi-static $d_{33} / d_{31}$ meter (Institute of Acoustics, Chinese Academy of Sciences, Beijing, China). Detailed electrical properties measurement procedures were described elsewhere [12].

\section{Results and discussion}

Figure 1 shows the XRD patterns of the Mn-doped $0.95 \mathrm{NBT}-0.05 \mathrm{BT}$ single crystals. To investigate the structural phase transition upon heating, two kinds of measurement apparatuses are used. Therefore, XRD patterns of Mn-doped 0.95NBT-0.05BT measured at room-temperature by the two apparatuses are given in the figure. It can be seen that the as-grown Mn-doped 0.95NBT-0.05BT single crystals exhibit phase-pure perovskite structure without detectable impurity, in which the marked impure X-ray reflections are caused by the scattering of the high-temperature apparatus. The relative intensity of this impurity tends to decrease with the increase of measurement temperature. Composition variation appears in the as-grown single crystals; however, within the detection sensitivity of the energy dispersive X-ray fluorescence elemental analyzer, the nominal composition can be used to represent the real composition of the single crystals.

Due to segregation during crystal growth, complex solid solution composition, and coexistence of different ferroelectric domains or polar states, the Mn-doped 0.95NBT$0.05 \mathrm{BT}$ single crystals exhibit complex crystal structure and can not be refined simply. Using the full diffraction profile fitting of the MDI Jade 5.0 software, crystal structure of Mn-doped 0.95NBT-0.05BT can be identified (Figure 1(b) shows the diffraction fitting of the $\{211\}$ diffraction reflection at $530^{\circ} \mathrm{C}$ as an example). Structural phase transition of the Mn-doped 0.95NBT-0.05BT single crystals upon heating can be seen in Figure 1 and Table 1. With the increase of temperature, the distortion of $\left[\mathrm{TiO}_{6}\right]$ octahedras decreases, leading to the change of dimension of crystal lattice ac- 

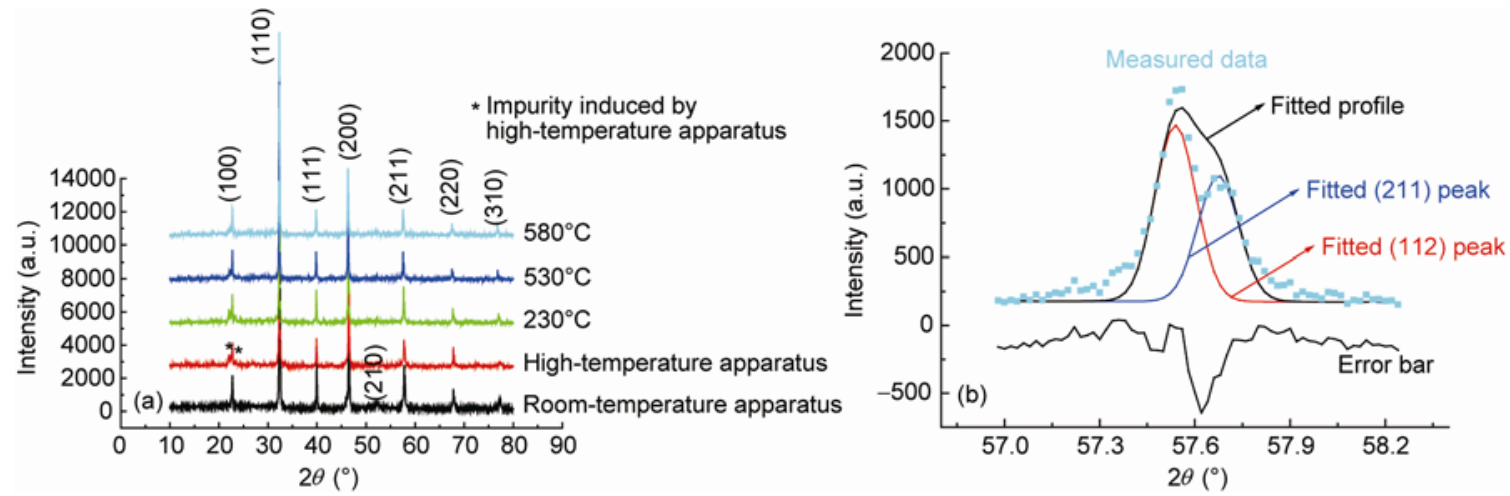

Figure 1 (a) XRD patterns of the Mn-doped 0.95NBT-0.05BT single crystal powder measured at different temperatures upon heating; (b) raw data, fitted profile and error bar of the $\{211\}$ diffraction reflection of the Mn-doped $0.95 \mathrm{NBT}-0.05 \mathrm{BT}$ single crystal powder at $530^{\circ} \mathrm{C}$.

Table 1 Cell parameters of the Mn-doped 0.95NBT-0.05BT single crystals at different temperatures

\begin{tabular}{cllcccc}
\hline Temperature $\left({ }^{\circ} \mathrm{C}\right)$ & $a=b(\AA)$ & \multicolumn{1}{c}{$c(\AA)$} & $\alpha\left({ }^{\circ}\right)$ & $\beta\left({ }^{\circ}\right)$ & $\gamma\left({ }^{\circ}\right)$ & Cell volume $\left(\AA^{3}\right)$ \\
\hline Room-temperature apparatus (RT) & $3.9058(28)$ & $3.9058(28)$ & $89.917(386)$ & $90.073(255)$ & $89.997(62)$ & 59.584 \\
High-temperature apparatus (HT) & $3.9054(3)$ & $3.9054(3)$ & $89.875(38)$ & $90.048(25)$ & $89.984(6)$ & 59.565 \\
$230(\mathrm{HT})$ & $3.9083(32)$ & $3.9183(46)$ & 90 & 90 & 90 & 59.852 \\
$530(\mathrm{HT})$ & $3.9200(32)$ & $3.9292(46)$ & 90 & 90 & 90 & 60.377 \\
$580(\mathrm{HT})$ & $3.9267(3)$ & $3.9267(3)$ & 90 & 90 & 90 & 60.543 \\
\hline
\end{tabular}

companied by the successive change of crystal structure. At room temperature, Mn-doped 0.95NBT-0.05BT can be identified as rhombohedral perovskite structure with apparent distortion, in which the cell parameters are refined as $a=b=c=3.9058 \AA, \alpha=89.917^{\circ}, \beta=90.073^{\circ}, \gamma=89.997^{\circ}$ and $V=59.584 \AA^{3}$. With the increase of temperature, high-angle diffraction reflections $\{200\},\{211\}$ and $\{220\}$ exhibit apparent tetragonal splitting, whereas other diffraction peaks exhibit broadened single peak, indicating that distorted rhombohedral phase and tetragonal phase coexist in the crystals between $230-530^{\circ} \mathrm{C}$. Above $530^{\circ} \mathrm{C}$ the Mn-doped $0.95 \mathrm{NBT}-0.05 \mathrm{BT}$ single crystals exhibit rather typical tetragonal perovskite structure, in which the tetragonality, i.e. the ratio of the cell parameters $c / a$, tends to increase with temperature. At $580^{\circ} \mathrm{C}$ all the diffraction peaks merge into sharp and symmetric single peak, indicating that Mn-doped 0.95NBT-0.05BT changes wholly into cubic structure, in which the cell parameters expand to $a=b=c=3.9267 \AA$, $\alpha=\beta=\gamma=90^{\circ}$, and $V=60.543 \AA^{3}$.

Therefore, at room temperature, the Mn-doped 0.95NBT$0.05 \mathrm{BT}$ single crystals locate on the rhombohedral side of the NBT-BT system, but very close to the MPB composition. Based on the bulk density measurement by Archimedes water immersion method and the XRD measurement result, the relative density of the Mn-doped $0.95 \mathrm{NBT}$ 0.05BT single crystals can be calculated, in which the relative density $=$ bulk density/theoretical density $=5.7423 / 5.9347$ $\times 100 \%=96.76 \%$. The as-grown Mn-doped 0.95NBT0.05BT single crystals exhibit high density, which approaches closely to its theoretical density.
Figure 2 shows 3-D graph of dielectric constantfrequency-DC voltage of the poled Mn-doped 0.95NBT$0.05 \mathrm{BT}$ crystal plates at room-temperature. The dielectric response peaks appearing at different frequencies relate to the different vibration modes of electromechanical coupling of the poled single crystals. Crystal orientation exerts great effects on the dielectric response character, in which the values of dielectric constant and the shape of the dielectric-frequency curves exhibit great differences. Such variation is considered as correlating with the differences of crystal symmetry deviation, ion spacing, electrostatic interaction and spontaneous polarization introduced by crystal orientation [13]. DC bias voltage exerts additional influence on the dielectric response character, which is considered as relating to the electric field induced micro-macro ferroelectric domain change and/or the structural ferroelectric phase transition. Usually, such mechanism can be confirmed by the dielectric-temperature spectrum measurement using the poled single crystals.

Figure 3 shows the temperature dependence of dielectric property of the poled Mn-doped 0.95NBT-0.05BT crystal plates. As compared to the undoped single crystals, the dielectric response peaks around the temperature of dielectric constant maximum $\left(T_{\mathrm{m}}\right)$ become broader and blurred, indicating the diffuse phase transition character of the ferroelectric phase-paraelectric phase transition. The dielectric frequency dispersion phenomenon becomes apparent below $T_{\mathrm{m}}$ and around the depolarization temperature $T_{\mathrm{d}}$, in which piezoelectric property of the NBT-based materials disappears when circumstance temperature reaches or exceeds 

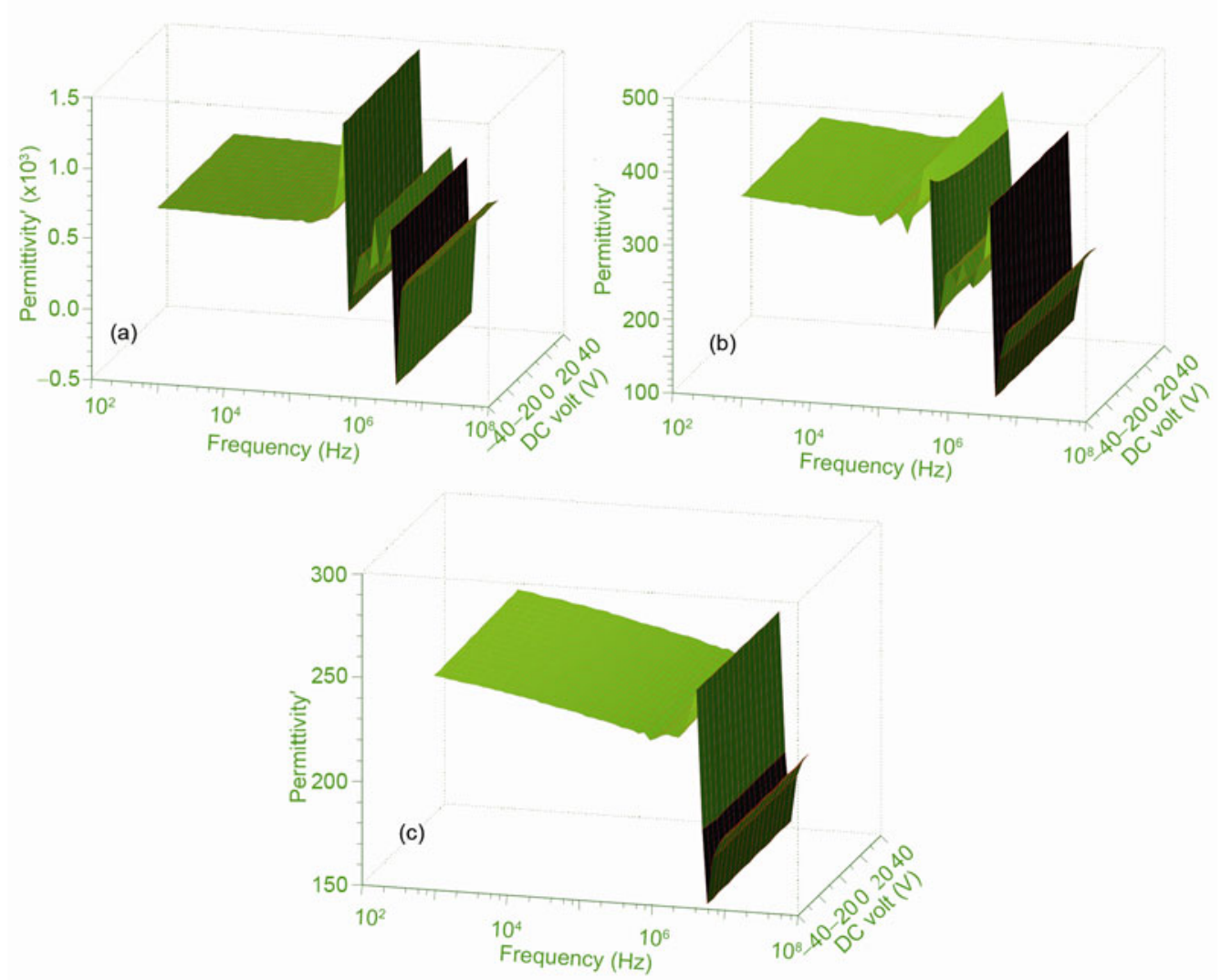

Figure 2 Frequency dependence of dielectric constant under different DC voltage of the poled Mn-doped 0.95NBT-0.05BT crystal plates with different orientations measured at room-temperature.

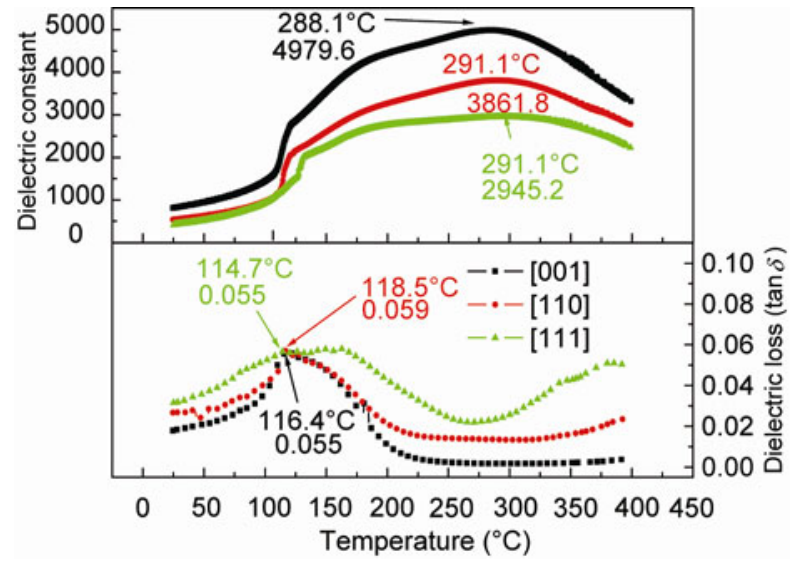

Figure 3 Temperature dependence of dielectric constant and loss tangent of the poled Mn-doped 0.95NBT-0.05BT crystal plates with different orientations measured at $10 \mathrm{kHz}$ upon heating.

this temperature [14]. After poling, the originally existed dielectric shoulder is induced into apparent dielectric anomaly around $115^{\circ} \mathrm{C}$, which is considered as correlating with the meta-stable ferroelectric phases, the orientation of ferroelectric domain and/or the macro-micro domain transition upon heating [8]. The additional electric field-induced dielectric anomaly provides good evidence for the above phenomena. Crystal orientation exerts great influences on die- lectric property, in which the different oriented Mn-doped 0.95NBT-0.05BT crystal plates exhibit different values of dielectric constant and loss tangent, and different dielectric response character.

The electric field induced domain change and/or the structural ferroelectric phase transition can be further confirmed by domain observation. Figure 4 shows the change of domain structure of the [001]-oriented Mn-doped $0.95 \mathrm{NBT}-0.05 \mathrm{BT}$ crystal plate upon rotation observed under crossed polarized-light. Since the light extinction rule of crystal structure under polarized-light is determined by crystallographic system symmetry, the structure character of poled crystals can be judged by this method. Although the Mn-doped 0.95NBT-0.05BT single crystals exist in distorted rhombohedral perovskite structure determined by X-ray powder diffraction, the multidomain configuration of the poled [001]-oriented crystal plate exhibits statistically $4 \mathrm{~mm}$ symmetry. By the way, the Mn-doped 0.95NBT-0.05BT single crystals exhibit complex domain structure, in which macro straight domain stripes and micro irregular-arranged domains coexist. Furthermore, substructures exist in the domain configuration, and different light extinction rule is observed in local area.

The existence of depolarization temperature $T_{\mathrm{d}}$ can be confirmed further by the measurement of the influences of environmental temperature on the piezoelectric properties. 

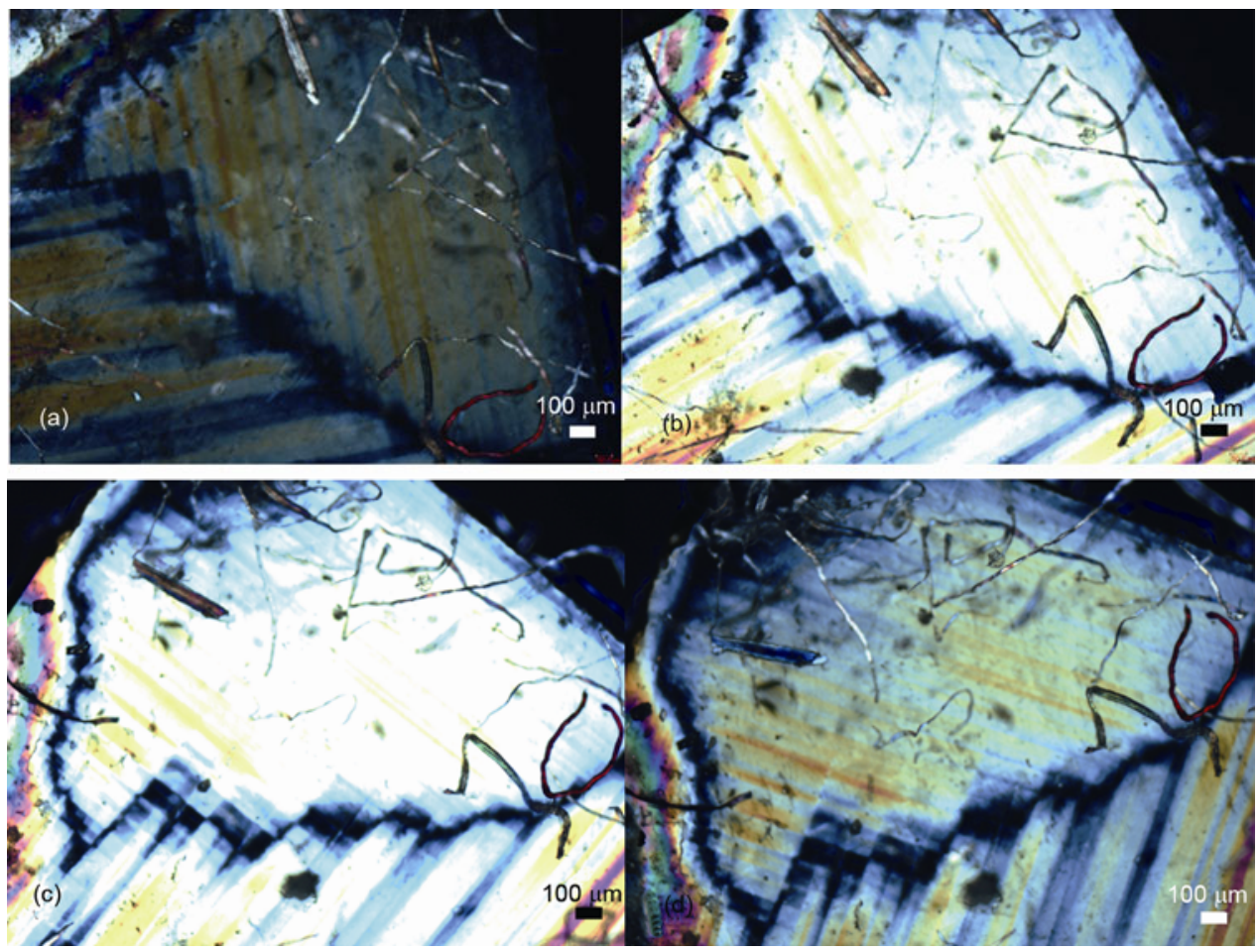

Figure 4 Domain configuration evolution of the poled [001]-oriented Mn-doped 0.95NBT-0.05BT crystal plate observed by polarizing microscope under crossed polarized-light. (a) Rotation $10^{\circ}$ from the light extinction site; (b) rotation $30^{\circ}$; (c) rotation $50^{\circ}$; (d) rotation $70^{\circ}$.

Due to Mn doping, the Mn-doped 0.95NBT-0.05BT single crystals exhibit large resistivity, being $6.151 \times 10^{15}, 8.236 \times$ $10^{15}$ and $2.987 \times 10^{16} \Omega \mathrm{m}$ for the [001]-, [110]- and [111]-oriented crystal plates, which are especially advantageous for the poling process of piezoelectric materials and are feasible to obtain excellent piezoelectric properties. Figure 5 shows the temperature dependence of piezoelectric constant $d_{33}$ and electromechanical coupling coefficient $K_{\mathrm{p}}$ of the Mn-doped 0.95NBT-0.05BT single crystals. As compared to piezoelectric ceramics [15], the variation of the values of $d_{33}$ is rather small, confirming partly the high-quality of the as-grown single crystals. The $d_{33}-T$ and $K_{\mathrm{p}}-T$ curves exhibit several regions with different slopes upon heating. The temperature of the dramatic decrease of the values of $d_{33}$ and $K_{\mathrm{p}}$ exhibit strong dependency on the crystal orientation, which is considered as correlating with the change of ferroelectric domain or the structural ferroelectric phase transition. Since the dielectric propertytemperature spectrum was measured by continuously linear increase of temperature, and the $d_{33}-T$ and $K_{\mathrm{p}}-T$ curves were measured by a quasi-static method, the temperature where the values of $d_{33}$ and $K_{\mathrm{p}}$ decrease greatly and the temperature $T_{\mathrm{d}}$ obtained by the dielectric anomaly in the dielectric loss- $T$ curve (Figure 3) are different. The depolarization temperature is very important for piezoelectric applications; therefore, the safe service temperature of the Mn-doped 0.95 NBT-0.05BT single crystal is below $110^{\circ} \mathrm{C}$.

For pyroelectric applications, low and stable values of

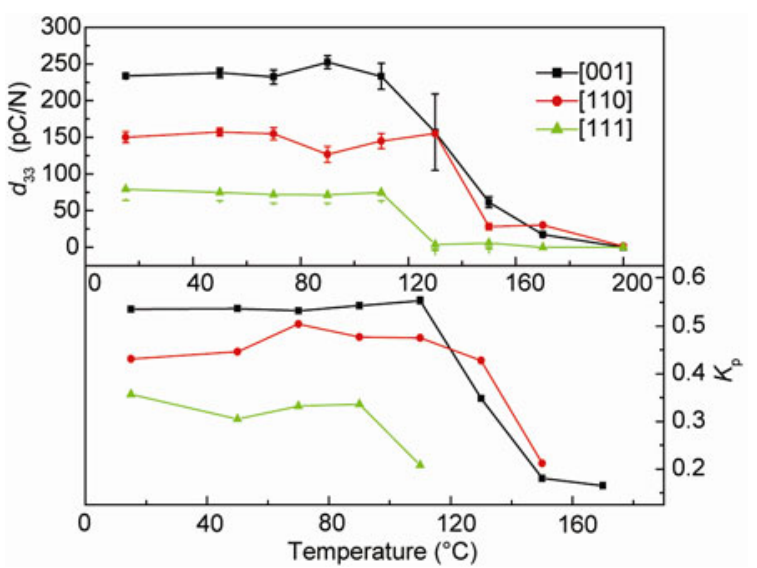

Figure 5 Temperature dependence of piezoelectric constant $d_{33}$ and radial-extension-vibration electromechanical coupling coefficient $K_{\mathrm{p}}$ of the Mn-doped 0.95NBT-0.05BT crystal plates with different orientations. The error bars of $d_{33}$ were obtained by measuring 5 samples and each sample selecting 9 positions.

specific heat $C_{\mathrm{p}}$ and dielectric constant are desirable to meet the requirements of response time and high responsivity. Figure 6 shows the temperature dependence of $C_{\mathrm{p}}$ and frequency dependence of dielectric constant of the Mn-doped $0.95 \mathrm{NBT}-0.05 \mathrm{BT}$ single crystals. The density of the Mn-doped 0.95NBT-0.05BT single crystals is $\sim 5.7423 \times 10^{3}$ $\mathrm{kg} / \mathrm{m}^{3}$, which remains almost unchangeable between 20 and $80^{\circ} \mathrm{C}$. Therefore, the volume specific heat $C_{\mathrm{v}}$ is $\sim 7.522 \times 10^{6}$ $\mathrm{J} /\left(\mathrm{m}^{3}{ }^{\circ} \mathrm{C}\right)$ at room-temperature, which increases $12.88 \%$ 




Figure 6 Temperature dependence of $C_{\mathrm{p}}$ of the Mn-doped $0.95 \mathrm{NBT}-0.05 \mathrm{BT}$ single crystal powder upon heating and frequency dependence of dielectric constant of the Mn-doped 0.95NBT-0.05BT crystal plates with different orientations at room-temperature.

between 20 and $80^{\circ} \mathrm{C}$. At $100 \mathrm{~Hz}$, dielectric constant and loss tangent are 846 and $0.0129,455$ and 0.0649 , and 472 and 0.0359 for the [001]-, [110]- and [111]-oriented Mn-doped 0.95 NBT-0.05BT crystal plates, respectively. The values of dielectric constant and loss tangent keep almost invariable between 100 and $2000 \mathrm{~Hz}$.

Figure 7 shows the pyroelectric properties of the Mn-doped 0.95 NBT-0.05BT single crystals. The pyroelectric properties exhibit great dependency on the crystal orientation, which is considered as relating to the differences of crystal symmetry deviation, complex domain configuration, complicated coexistent ferroelectric phases and spontaneous polarization direction. At room temperature, the measured pyroelectric coefficients are 834.1, 798.1 and $691.1 \mu \mathrm{C} /\left(\mathrm{K} \mathrm{m}^{2}\right)$ for the [001]-, [110]- and [111]-oriented

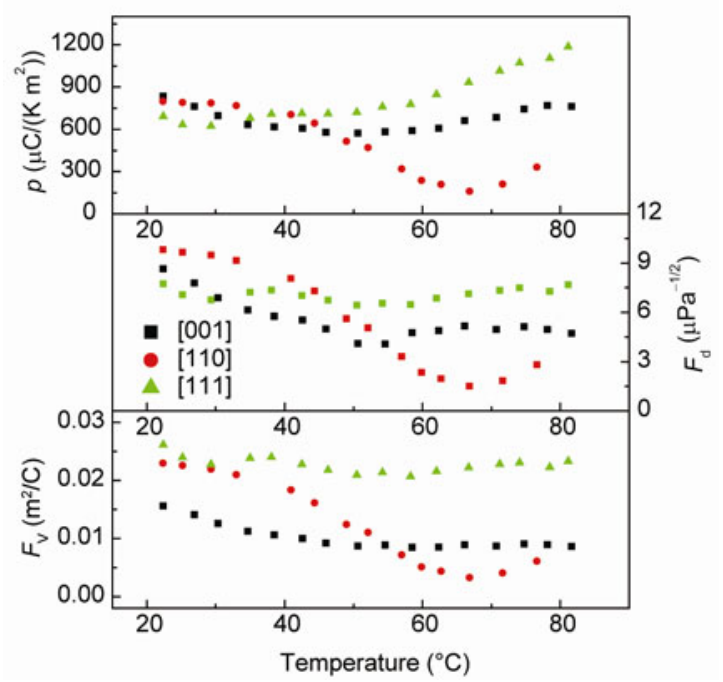

Figure 7 Temperature dependence of pyroelectric coefficient $p$, and figures of merit $F_{\mathrm{d}}$ and $F_{\mathrm{v}}$ of the Mn-doped $0.95 \mathrm{NBT}-0.05 \mathrm{BT}$ crystal plates with different orientations.
Mn-doped 0.95NBT-0.05BT crystal plates, respectively, greatly larger than that of $\mathrm{LiTaO}_{3}\left(230 \mu \mathrm{C} /\left(\mathrm{K} \mathrm{m}^{2}\right)\right)$ [16]. With the increase of temperature, the pyroelectric properties of the different oriented crystal plates exhibit different change character. Whatever increase or decrease of the values of the pyroelectric coefficient around end temperatures of measurement, such change is considered as correlating with the change of ferroelectric domain or the structural ferroelectric phase transition. From room temperature to $80^{\circ} \mathrm{C}$, the pyroelectric coefficients decrease $8.79 \%, 58.48 \%$, and increase $59.69 \%$ for the [001]-, [110]- and [111]oriented Mn-doped 0.95NBT-0.05BT crystal plates, respectively, which is slightly large for practical applications. Further work on improving temperature stability of the pyroelectric property deserves special attention. Pyroelectric figures of merit were calculated based on the above experimental results, for current responsivity $F_{\mathrm{i}}=p / C_{\mathrm{v}}$, for voltage responsivity $F_{\mathrm{v}}=p /\left(C_{\mathrm{v}} \varepsilon_{0} \varepsilon_{\mathrm{r}}\right)$, and for detectivity $F_{\mathrm{d}}=p /\left[C_{\mathrm{v}}\left(\varepsilon_{0} \varepsilon_{\mathrm{r}} \tan \delta\right)^{1 / 2}\right]$, in which $p, C_{\mathrm{v}}, \varepsilon_{0}, \varepsilon_{\mathrm{r}}$ and $\tan \delta$ are the pyroelectric coefficient, volume specific heat, permittivity of free space $\left(8.854 \times 10^{-12} \mathrm{~F} / \mathrm{m}\right)$, relative dielectric constant, and dielectric loss, respectively [16]. At room temperature, $F_{\mathrm{d}}$ is $8.639,9.817$ and $7.739 \mu \mathrm{Pa}^{-1 / 2}$, and $F_{\mathrm{v}}$ is $0.0156,0.0230$ and $0.0262 \mathrm{~m}^{2} / \mathrm{C}$ for the [001]-, [110]- and [111]-oriented Mn-doped 0.95NBT-0.05BT crystal plates, respectively. The values of $F_{\mathrm{d}}$ and $F_{\mathrm{v}}$ remain almost unchangeable over the frequency range $100-2000 \mathrm{~Hz}$, whereas these values exhibit similar change character to that of $p$ over the temperature range measured. Significant fundamental electrical properties for pyroelectric applications of the Mn-doped 0.95NBT-0.05BT single crystals are summarized in Table 2. Although volume specific heat of $\mathrm{Mn}$ doped $0.95 \mathrm{NBT}-0.05 \mathrm{BT}$ is relatively large, enhanced pyroelectric properties can be obtained in the lead-free single crystals, which will lead to high responsivity and detectivity for pyroelectric applications.

Infrared and Raman spectroscopy provide useful information on atomic separation, spatial arrangement of atoms, inter-atom force and crystal structure. Figure 8 shows FT-IR spectra of the Mn-doped 0.95NBT-0.05BT single crystals, in which the available experimental range is $4000-400 \mathrm{~cm}^{-1}$ and $4000-650 \mathrm{~cm}^{-1}$ for transmission mode and reflection mode, respectively. Due to the complex cation substitution of the A-site of the perovskite structure, the location of the infrared absorption band shifts slightly to low frequency, and the absorption band widens greatly and exhibits strong asymmetry of Mn-doped $0.95 \mathrm{NBT}-0.05 \mathrm{BT}$ as compared to those of simple perovskite $\mathrm{PbTiO}_{3}$ [17]. In the transmission mode, two absorption bands tend to appear. The higher asymmetric frequency band, $v_{1}$, with an apparent highfrequency tail and a center of $630.6 \mathrm{~cm}^{-1}$, can be assigned to the $\left[\mathrm{Na}_{1 / 2} \mathrm{Bi}_{1 / 2}\right] / \mathrm{Ti}-\mathrm{O}$ "stretching" normal vibration. The lower frequency band, $v_{2}$, extending from $472.5 \mathrm{~cm}^{-1}$ 
Table 2 Important electrical properties of the Mn-doped 0.95NBT-0.05BT single crystals for pyroelectric devices at room temperature

\begin{tabular}{cccccccccc}
\hline Crystal orientation & $\begin{array}{c}\varepsilon_{\mathrm{r}} \\
(100 \mathrm{~Hz})\end{array}$ & $\begin{array}{c}\tan \delta \\
(100 \mathrm{~Hz})\end{array}$ & $\begin{array}{c}P_{\mathrm{r}} \\
\left(\mu \mathrm{C} / \mathrm{cm}^{2}\right)\end{array}$ & $\begin{array}{c}E_{\mathrm{c}} \\
(\mathrm{kVV} / \mathrm{mm})\end{array}$ & $\begin{array}{c}d_{33} \\
(\mathrm{pC} / \mathrm{N})\end{array}$ & $\begin{array}{c}p \\
\left(\mu \mathrm{C} / \mathrm{K} \mathrm{m}^{2}\right)\end{array}$ & $\begin{array}{c}F_{\mathrm{i}} \\
(\mathrm{pm} / \mathrm{V})\end{array}$ & $\begin{array}{c}F_{\mathrm{v}} \\
\left(\mathrm{m}^{2} / \mathrm{C}\right)\end{array}$ & $\begin{array}{c}F_{\mathrm{d}} \\
\left.(\mu \mathrm{Pa})^{-1 / 2}\right)\end{array}$ \\
\hline$[001]$ & 846 & 0.0129 & 7.52 & 2.047 & 233.4 & 834.1 & 110.9 & 0.0156 & 8.639 \\
{$[110]$} & 455 & 0.0649 & 9.59 & 2.951 & 150.2 & 798.1 & 106.1 & 0.0230 & 9.817 \\
{$[111]$} & 472 & 0.0359 & 20.92 & 2.984 & 79.2 & 691.1 & 91.9 & 0.0262 & 7.739 \\
\hline
\end{tabular}

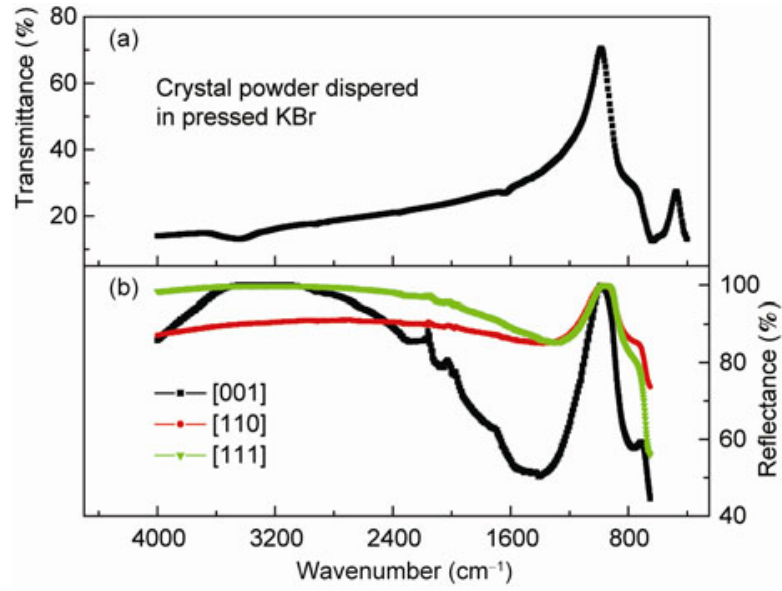

Figure 8 FT-IR spectra of Mn-doped 0.95NBT-0.05BT. (a) Absorption spectrum of the Mn-doped 0.95NBT-0.05BT crystal powder dispersed in pressed $\mathrm{KBr}$ disk; (b) reflection spectra of the Mn-doped 0.95NBT-0.05BT crystal plates with different orientations.

and the band center being beyond the available experiment range, can be assigned to the $\left[\mathrm{Na}_{1 / 2} \mathrm{Bi}_{1 / 2}\right] / \mathrm{Ti}-\mathrm{O}$ "bending" normal vibration [17]. In the reflection mode, the infrared spectrum correlates with crystal absorption and crystal surface reflection, whereas the crystal surface reflectivity exerts little effect on the transmission mode. The location of the infrared absorption band tends to move to high frequency, whereas the extinction frequency and the center of the higher frequency band, $v_{1}$, and the lower frequency band, $v_{2}$, cannot be observed by our experimental. An additional absorption band appears in all the crystal plates. Crystal orientations exert great effects on the location of absorption band, the absorption intensity and the shape of infrared absorption band; therefore, the infrared spectra exhibit strong dependency on local crystal structure and/or special domain configuration.

Figure 9 shows room-temperature Raman spectra of the Mn-doped 0.95NBT-0.05BT single crystals, which is sensitive to short-range ordering non-uniform distortions of crystal lattice. Crystal orientation exerts great influence on the Raman spectra due to the differences of ionic separation distance, atomic interaction, cation coordination circumstance, domain configuration and local crystal structure. Three strong Raman bands appear in all the crystal plates, in which the shape, positions, intensity and bandwidth of the peaks exhibit great differences on the crystal orientations. The diffused Raman bands centered around 300, 560

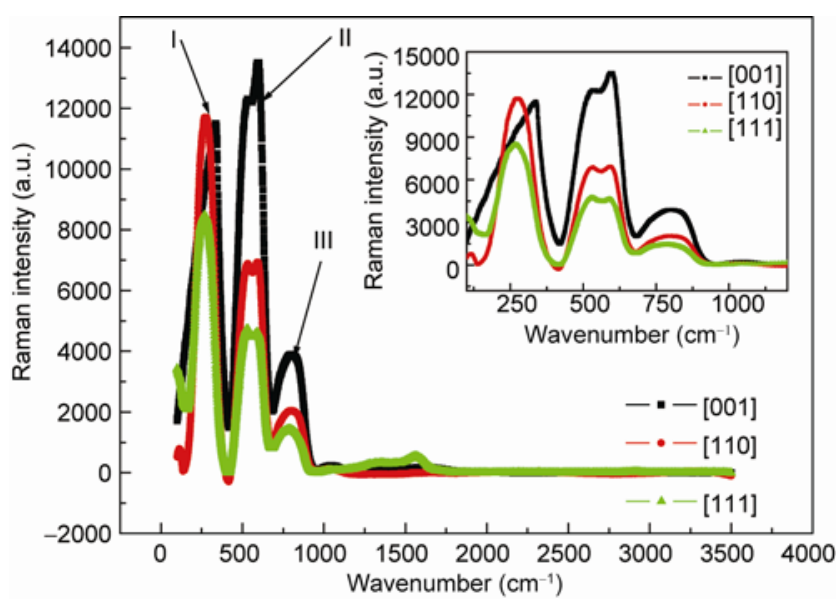

Figure 9 Raman spectra of the Mn-doped 0.95NBT-0.05BT crystal plates with different orientations at room temperature.

and $800 \mathrm{~cm}^{-1}$ can be attributed to $\mathrm{F} 2 \mathrm{~g}\left[\mathrm{TiO}_{6}\right]$ bending vibration, $\mathrm{A} 1 \mathrm{~g}\left[\mathrm{TiO}_{6}\right]$ stretching vibration and "soft mode" mixed by bending and stretching vibrations $[5,7,18]$. Fine Raman spectroscopy research indicates that the $\left[\mathrm{TiO}_{6}\right]$ octahedron vibrations are composed of six species of vibration modes [18]. Such Raman spectra exhibit great differences from those of the bulk polycrystalline $0.95 \mathrm{NBT}-0.05 \mathrm{BT}$ ceramics [5] and the 0.95NBT-0.05BT single crystals fabricated by the solid state single crystal growth technique [7], which can be attributed to the difference of crystalline type and the distortion of the perovskite structure induced by Mn-doping.

\section{Conclusions}

The 0.14 at $\%$ Mn-doped 0.95NBT-0.05BT lead-free single crystals exhibit distorted rhombohedral perovskite structure, which undergoes successive structural phase transitions upon heating. Between 230 and $530^{\circ} \mathrm{C}$ rhombohedral phase and tetragonal phase coexist in the single crystals, which change wholly into cubic structure at $580^{\circ} \mathrm{C}$. The Mn-doped $0.95 \mathrm{NBT}-0.05 \mathrm{BT}$ single crystals exhibit high density, being $96.76 \%$ of the theoretical density. Crystal orientations and polarization exert great effects on domain configuration, optical and electrical properties. Polarization induces apparent dielectric anomaly accompanied by the variation of dielectric response character, which can be attributed to the orientation of ferroelectric domain and/or the macro-micro 
domain transition. The Mn-doped 0.95NBT-0.05BT single crystals exhibit statistically $4 \mathrm{~mm}$ symmetry although complex domain configuration exists. The infrared-absorption band occurred around $630.6 \mathrm{~cm}^{-1}$ and the diffused Raman bands centered around $300 \mathrm{~cm}^{-1}, 560 \mathrm{~cm}^{-1}$ and $800 \mathrm{~cm}^{-1}$ are observed by FT-IR and Raman spectroscopy, which exhibit great dependency on crystal orientations. [001]-oriented Mn-doped 0.95NBT-0.05BT crystal plates exhibit excellent electrical properties, in which the values of $d_{33}$ is 233.4 $\mathrm{pC} / \mathrm{N}, K_{\mathrm{p}}$ is $0.536, P_{\mathrm{r}}$ is $7.52 \mu \mathrm{C} / \mathrm{cm}^{2}, E_{\mathrm{c}}$ is $2.047 \mathrm{kV} / \mathrm{mm}, p$ is $834.1 \mu \mathrm{C} /\left(\mathrm{K} \mathrm{m}^{2}\right), F_{\mathrm{i}}$ is $110.9 \mathrm{pm} / \mathrm{V}, F_{\mathrm{d}}$ is $8.639 \mu \mathrm{Pa}^{-1 / 2}$ and $F_{\mathrm{v}}$ is $0.0156 \mathrm{~m}^{2} / \mathrm{C}$.

This work was supported by the National Natural Science Foundation of China (51242006), the Qing Lan Project, the Key Laboratory of Inorganic Function Material and Device, Chinese Academy of Sciences (KLIFMD-2011-02) and the Priority Academic Program Development of Jiangsu Higher Education Institutions.

1 Saito Y, Takao H, Tani T, et al. Lead-free piezoceramics. Nature, 2004, 432: 84-87

2 Wang $\mathrm{P}, \mathrm{Li} \mathrm{Y}, \mathrm{Lu} \mathrm{Y}$. Enhanced piezoelectric properties of $\left(\mathrm{Ba}_{0.85} \mathrm{Ca}_{0.15}\right)\left(\mathrm{Ti}_{0.9} \mathrm{Zr}_{0.1}\right) \mathrm{O}_{3}$ lead-free ceramics by optimizing calcination and sintering temperature. $\mathbf{J}$ Eur Ceram Soc, 2011, 31: 2005-2012

3 Park H Y, Seo I T, Choi J H, et al. Low-temperature sintering and piezoelectric properties of $\mathrm{Na}_{0.5} \mathrm{~K}_{0.5} \mathrm{NbO}_{3}$ lead-free piezoelectric ceramics. J Am Ceram Soc, 2010, 93: 36-39

4 Takenaka T, Maruyama K I, Sakata K. $\left(\mathrm{Bi}_{0.5} \mathrm{Na}_{0.5}\right) \mathrm{TiO}_{3}-\mathrm{BaTiO}_{3}$ system for lead-free piezoelectric ceramics. Jpn J Appl Phys, 1991, 30: 2236-2239

5 Rout D, Moon K S, Rao V S, et al. Study of the morphotrophic phase boundary in the lead-free $\mathrm{Na}_{1 / 2} \mathrm{Bi}_{1 / 2} \mathrm{TiO}_{3}-\mathrm{BaTiO}_{3}$ system by Raman spectroscopy. J Ceram Soc Jpn, 2009, 117: 797-800

6 Zhang Q, Zhang Y, Wang F, et al. Growth and electric properties of
0.96 $\mathrm{Na}_{0.5} \mathrm{Bi}_{0.5} \mathrm{TiO}_{3}-0.04 \mathrm{BaTiO}_{3}$ single crystal. J Cryst Growth, 2010, 312: 457-460

7 Moon K S, Rout D, Lee $\mathrm{H} \mathrm{Y}$, et al. Solid state growth of $\mathrm{Na}_{1 / 2} \mathrm{Bi}_{1 / 2} \mathrm{TiO}_{3}-\mathrm{BaTiO}_{3}$ single crystals and their enhanced piezoelectric properties. J Cryst Growth, 2011, 317: 28-31

8 Zhao X, Fang B, Cao H, et al. Dielectric and piezoelectric performance of PMN-PT single crystals with compositions around the MPB: Influence of composition, poling field and crystal orientation. Mater Sci Eng B, 2002, 96: 254-262

$9 \mathrm{Fu} \mathrm{H}$, Cohen R E. Polarization rotation mechanism for ultrahigh electromechanical response in single-crystal piezoelectrics. Nature, 2000. 403: 281-283

10 Cao H, Bai F, Wang N, et al. Intermediate ferroelectric orthorhombic and monoclinic $\mathrm{MB}$ phases in [110] electric-field-cooled $\mathrm{Pb}$ $\left(\mathrm{Mg}_{1 / 3} \mathrm{Nb}_{2 / 3}\right) \mathrm{O}_{3}-30 \% \mathrm{PbTiO}_{3}$ crystals. Phys Rev B, 2005, 72: 064104

11 Chiang Y M, Farrey G W, Soukhojak A N. Lead-free high-strain single-crystal piezoelectrics in the alkaline-bismuth-titanate perovskite family. Appl Phys Lett, 1998, 73: 3683-3685

12 Fang B, Qian K, Miao F, et al. Structural, optical and improved electrical properties of relaxor-based single crystals after poling. J Am Ceram Soc, 2012, 95: 1949-1954

13 Liu W, Wang G, Cao S, et al. The phase transition behavior of $(1-x) \mathrm{Pb}\left(\mathrm{Sc}_{0.5} \mathrm{Ta}_{0.5}\right) \mathrm{O}_{3}-(x) \mathrm{PbHfO}_{3}$ ceramics. J Am Ceram Soc, 2011, 94: 2530-2534

14 Sun R, Zhao X, Zhang Q, et al. Growth and orientation dependence of electrical properties of $0.92 \mathrm{Na}_{0.5} \mathrm{Bi}_{0.5} \mathrm{TiO}_{3}-0.08 \mathrm{~K}_{0.5} \mathrm{Bi}_{0.5} \mathrm{TiO}_{3}$ leadfree piezoelectric single crystal. J Appl Phys, 2011, 109: 124113

15 Fang B, Du Q, Wu D, et al. Structural and electrical properties of $0.56 \mathrm{~Pb}\left(\mathrm{Ni}_{1 / 3} \mathrm{Nb}_{2 / 3}\right) \mathrm{O}_{3}-0.10 \mathrm{~Pb}\left(\mathrm{Zn}_{1 / 3} \mathrm{Nb}_{2 / 3}\right) \mathrm{O}_{3}-0.34 \mathrm{PbTiO}_{3}$ ceramics prepared by different ceramic processings. Ceram Inter, 2011, 37: 707-713

16 Tang Y, Zhao X, Feng X, et al. Pyroelectric properties of [111]oriented $\mathrm{Pb}\left(\mathrm{Mg}_{1 / 3} \mathrm{Nb}_{2 / 3}\right) \mathrm{O}_{3}-\mathrm{PbTiO}_{3}$ crystals. Appl Phys Lett, 2005, 86: 082901

17 Last J T. Infrared-absorption studies on barium titanate and related materials. Phys Rev, 1957, 105: 1740-1750

18 Chan I H, Sun C T, Houng M P, et al. Sb doping effects on the piezoelectric and ferroelectric characteristics of lead-free $\mathrm{Na}_{0.5} \mathrm{~K}_{0.5^{-}}$ $\mathrm{Nb}_{1-x} \mathrm{Sb}_{x} \mathrm{O}_{3}$ piezoelectric ceramics. Ceram Inter, 2011, 37: 20612068

Open Access This article is distributed under the terms of the Creative Commons Attribution License which permits any use, distribution, and reproduction in any medium, provided the original author(s) and source are credited. 